\title{
Generalized Linguistic Hesitant Intuitionistic Fuzzy Hybrid Aggregation Operators
}

\author{
Wei Yang, Jiarong Shi, and Yongfeng Pang \\ Department of Mathematics, School of Science, Xian University of Architecture and Technology, Xian 710055, China \\ Correspondence should be addressed to Wei Yang; yangweipyf@163.com
}

Received 15 January 2015; Accepted 28 May 2015

Academic Editor: Emiliano Mucchi

Copyright (C) 2015 Wei Yang et al. This is an open access article distributed under the Creative Commons Attribution License, which permits unrestricted use, distribution, and reproduction in any medium, provided the original work is properly cited.

\begin{abstract}
Some hybrid aggregation operators have been developed based on linguistic hesitant intuitionistic fuzzy information. The generalized linguistic hesitant intuitionistic fuzzy hybrid weighted averaging (GLHIFHWA) operator and the generalized linguistic hesitant intuitionistic fuzzy hybrid geometric mean (GLHIFHGM) operator are defined. Some special cases of the new aggregation operators are studied and many existing aggregation operators are special cases of the new operators. A new multiple attribute decision making method based on the new aggregation operators is proposed and a practical numerical example is presented to illustrate the feasibility and practical advantages of the new method.
\end{abstract}

\section{Introduction}

Fuzziness and uncertainty exist extensively in decision making process. Many useful tools have been developed to model fuzzy and uncertain information such as fuzzy set [1], intuitionistic fuzzy set [2], interval-valued intuitionistic fuzzy set [3], linguistic arguments [4, 5], and hesitant fuzzy set $[6,7]$. Hesitant fuzzy set was developed by Torra and Narukawa, which is the generalization of fuzzy set, and permits the membership having a set of possible values. The envelope of the hesitant fuzzy set is the intuitionistic fuzzy set. Hesitant fuzzy set has been studied and applied extensively since it can model fuzzy and uncertain information more accurately and flexibly. Some aggregation operators have been developed. Xia and $\mathrm{Xu}$ [8] discuss the relationship between the hesitant fuzzy set and intuitionistic fuzzy set. Zhu et al. [9] presented the hesitant fuzzy geometric Bonferroni mean and the hesitant fuzzy Choquet geometric Bonferroni mean. Wei [10] defined some prioritized aggregation operators for aggregating hesitant fuzzy information. Zhang et al. [11] presented induced generalized hesitant fuzzy ordered weighted averaging (IGHFOWA) operator and induced generalized hesitant fuzzy ordered weighted geometric (IGHFOWG) operator. A family of hesitant fuzzy Hamacher operators is proposed for aggregating hesitant fuzzy information based on the Hamacher t-norm and t-conorm [12]. Zhang [13] defined some hesitant fuzzy power aggregation operators. Some distance measures, similarity measures, and correlation measures have been defined. Xu and Xia [14] defined the distance and correlation measures for hesitant fuzzy information. The relationship between the entropy and the similarity measure and the distance measure for hesitant fuzzy sets (HFSs) and interval-valued hesitant fuzzy sets (IVHFSs) has been studied by Farhadinia [15]. Ye [16] proposes a correlation coefficient between dual hesitant fuzzy set as a new extension of existing correlation coefficients for hesitant fuzzy sets and intuitionistic fuzzy sets. Some multiple attribute decision making methods have been generalized to accommodate hesitant fuzzy information. Chen and Xu [17] developed a new approach, named HF-ELECTRE II approach, that combines the idea of HFSs with the ELECTRE II method. Zhang and Wei [18] extended the VIKOR to hesitant fuzzy set setting. Hesitant fuzzy set has been generalized to accommodate interval values [19], triangular fuzzy values [20], and linguistic arguments [21-23].

Though many useful methods have been developed for hesitant fuzzy information, there are still many decision making problems cannot be solved properly by using the existing methods since decision problems have become more and more complex with the developing of science and technology. Linguistic models have been developed and used extensively in decision problems since fuzzy nature of human 
thinking can be reflected. In evaluating process, decision makers express some hesitation, which can be modeled effectively by intuitionistic fuzzy values. For example, in evaluating performance of a candidate, one expert thinks he belongs to the degree of "good $\left(s_{7}\right)$ " which is $(0.7,0.2)$ and the degree of "very good $\left(s_{8}\right)$ " is $(0.6,0.3)$. The other expert think he belongs to the degree of "slight good $\left(s_{6}\right)$ " which is $(0.8,0.1)$ and the degree "good $\left(s_{7}\right)$ " is $(0.6,0.2)$. They cannot persuade each other and the evaluation values can be represented as $\check{h}=\left\{\left(s_{6},(0.8,0.1)\right),\left(s_{7},(0.7,0.2),(0.6,0.2)\right),\left(s_{8},(0.6,0.3)\right)\right\}$. The information is defined as linguistic hesitant intuitionistic fuzzy information. In this paper, we develop the linguistic hesitant intuitionistic fuzzy set based on the linguistic term set and intuitionistic fuzzy set. We define the generalized linguistic hesitant intuitionistic fuzzy hybrid weighted averaging (GLHIFHWA) operator. A wide range of aggregation operators are special cases of the GLHIFHWA operator, including generalized linguistic hesitant intuitionistic fuzzy ordered weighted averaging (GLHIFOWA) operator, the generalized linguistic hesitant intuitionistic fuzzy weighted averaging (GLHIFWA) operator, the linguistic hesitant intuitionistic fuzzy hybrid weighted averaging (LHIFHWA) operator, and the linguistic hesitant intuitionistic fuzzy hybrid weighted quadratic averaging (LHIFHWQA) operator. We define the generalized linguistic hesitant intuitionistic fuzzy hybrid geometric mean (GLHIFHGM) operator. Many exiting aggregation operators are special cases of the GLHIFHGM operator including the generalized linguistic hesitant hybrid geometric mean (GLHHGM) operator, the linguistic hesitant intuitionistic fuzzy hybrid geometric mean (LHIFHGM) operator, the linguistic hesitant intuitionistic fuzzy hybrid quadratic geometric mean (LHIFHQGM) operator, and the generalized linguistic hesitant intuitionistic fuzzy ordered geometric mean (GLHIFOGM) operator. Based on the two new aggregation operators, we develop a new multiple attribute decision making method. Finally, we apply the new method to a numerical example to illustrate its feasibility and practical advantages.

This paper is organized as follows. In Section 2, we first briefly introduce the concepts of hesitant fuzzy sets. Then, we present linguistic hesitant intuitionistic fuzzy set and some operational laws of the linguistic hesitant intuitionistic fuzzy elements. In Section 3, we present the GLHIFHWA operator and the GLHIFHGM operator. Some special cases of the new aggregation operators are studied. In Section 4, a new multiple attribute decision making method based on the new aggregation operators is developed. In Section 5, a numerical example is presented to illustrate the new method. The conclusions are presented in the last section.

\section{Linguistic Hesitant Intuitionistic Fuzzy Term Set}

Definition 1 (see [7]). Let $X$ be a reference set. An HFS $A$ on $X$ is a function $h$ that returns a subset of values in $[0,1]$ when it is applied to $X$ :

$$
A=\left\{\left\langle x, h_{A}(x)\right\rangle \mid x \in X\right\}
$$

where $h_{A}(x)$ is a set of some different values in $[0,1]$, representing the possible membership degrees of the element $x \in X$ to $A . h_{A}(x)$ is called a hesitant fuzzy element (HFE).

Linguistic arguments are used extensively since uncertainty of information and fuzzy nature of human thinking can be effectively modeled. Suppose that $S=\left\{s_{i} \mid i=1, \ldots, g\right\}$ is a finite and totally ordered discrete term set, where $s_{i}$ represents a possible value for a linguistic variable. A set of nine terms $S$ [24] can be expressed as follows:

$$
\begin{aligned}
S= & \left\{s_{1}=\text { extremely poor, } s_{2}=\text { very poor, } s_{3}\right. \\
& =\text { poor, } s_{4}=\text { slightly poor, } s_{5}=\text { fair, } s_{6} \\
& =\text { slightly good, } s_{7}=\text { good, } s_{8}=\text { very good, } s_{9} \\
& =\text { extremely good }\} .
\end{aligned}
$$

In order to preserve all the information, the discrete linguistic term set $S$ can be extended to a continuous one $\bar{S}=\left\{s_{\alpha} \mid s_{0} \leq s_{\alpha} \leq s_{g}, \alpha \in[0, g]\right\}$. If $s_{\alpha} \in S$, it is called the original linguistic term and it is called virtual linguistic term otherwise.

The distance measure between $s_{\alpha}$ and $s_{\beta}$ can be defined as

$$
d\left(s_{\alpha}, s_{\beta}\right)=\frac{1}{g}|\alpha-\beta|,
$$

where $s_{\alpha}, s_{\beta} \in \bar{S}$ are two linguistic terms.

As generalization of hesitant fuzzy sets (HFSs), Zhang and Wu [25] develop hesitant fuzzy linguistic term sets (HFLSs), in which a linguistic variable has several linguistic terms.

Definition 2. Let $X$ be a reference set and let $\bar{S}=\left\{s_{\alpha} \mid\right.$ $\left.s_{0} \leq s_{\alpha} \leq s_{g}\right\}$ be a linguistic term set. A hesitant fuzzy linguistic term set (HFLS) $\bar{A}$ on $X$ is an ordered finite subset of consecutive linguistic terms of $\bar{S}$

$$
\bar{A}=\left\{\left\langle x_{i}, \bar{h}_{\bar{A}}\left(x_{i}\right)\right\rangle \mid x_{i} \in X, i=1,2, \ldots, n\right\},
$$

where $\bar{h}_{\bar{A}}\left(x_{i}\right): X \rightarrow \bar{S}$ denotes all the possible linguistic evaluation values of element $x_{i} \in X$. For convenience, we call $\bar{h}_{\bar{A}}\left(x_{i}\right)$ a hesitant fuzzy linguistic element (HFLE), which can be represented as

$$
\bar{h}_{\bar{A}}\left(x_{i}\right)=\left\{s_{i} \mid s_{i} \in \bar{h}_{\bar{A}}\left(x_{i}\right)\right\}
$$

and here $s_{i}$ is a linguistic argument.

In decision making process, the expert may think some linguistic term can be used when evaluating, but he/she may have hesitation. Intuitionistic fuzzy value can be used to model hesitation existing in decision making process. The hesitant intuitionistic fuzzy linguistic term set can be defined by using the linguistic arguments and intuitionistic fuzzy values, which can be defined as follows.

Definition 3. Let $X=\left\{x_{1}, x_{2}, \ldots, x_{n}\right\}$ be a reference set and let $\bar{S}=\left\{s_{\alpha} \mid s_{0} \leq s_{\alpha} \leq s_{g}\right\}$ be a linguistic term set. 
A linguistic hesitant intuitionistic fuzzy term set (LHIFS) $\check{A}$ on $X$ is defined as

$$
\check{A}=\left\{\left\langle x_{i}, \check{h}_{\check{A}}\left(x_{i}\right)\right\rangle \mid x_{i} \in X, i=1,2, \ldots, n\right\},
$$

where $\check{h}_{\check{A}}\left(x_{i}\right): X \rightarrow H$ denotes all possible intuitionistic fuzzy linguistic evaluation values of element $x_{i} \in X$. For convenience, we call $\check{h}_{\check{A}}\left(x_{i}\right)$ a linguistic hesitant intuitionistic fuzzy element (LHIFE), which can be represented as

$$
\begin{aligned}
\check{h}_{\check{A}} & \left(x_{i}\right) \\
\quad & =\left\{\left(s_{\theta(i)}, \ln \left(s_{\theta(i)}\right)\right) \mid\left(s_{\theta(i)}, \operatorname{lh}\left(s_{\theta(i)}\right)\right) \in \check{h}_{\check{A}}\left(x_{i}\right)\right\},
\end{aligned}
$$

where $s_{\theta(i)}$ is a linguistic argument and $\operatorname{lh}\left(s_{\theta(i)}\right)$ is the set of intuitionistic fuzzy membership values $s_{\theta(i)}$ satisfying $x_{i}$. $\left(s_{\theta(i)}, \operatorname{lh}\left(s_{\theta(i)}\right)\right)$ is the linguistic intuitionistic fuzzy element (LIFE). $H$ is the set of all LHIFEs.

If all intuitionistic fuzzy membership $\left(\alpha_{i}, \beta_{i}\right)$ reduces to $\left(\mu_{i}, 1-\mu_{i}\right)$ in LIFEs, then LHIFE reduces to the linguistic hesitant fuzzy element (LHFE) as $h_{\check{A}}\left(x_{i}\right)=\left\{\left(s_{\theta(i)}, m\left(s_{\theta(i)}\right)\right) \mid\right.$ $\left.\left(s_{\theta(i)}, m\left(s_{\theta(i)}\right)\right) \in h_{\check{A}}\left(x_{i}\right)\right\}$, where $m\left(s_{\theta(i)}\right)$ is the set of fuzzy memberships with $s_{\theta(i)}$ satisfying the attribute $x_{i}$. If all intuitionistic fuzzy membership $\left(\alpha_{i}, \beta_{i}\right)$ reduces to $(1,0)$, then the LHIFE reduces to the hesitant fuzzy linguistic element (HFLE).

Definition 4. Let $\check{h}_{1}, \check{h}_{2}$, and $\check{h}$ be LHIFEs, $\lambda>0$. Consider that $a_{k}=\left(s_{\theta(k)}, \operatorname{lh}\left(s_{\theta(k)}\right)\right) \in \check{h}, a_{i}=\left(s_{\theta(i)}, \operatorname{lh}\left(s_{\theta(i)}\right)\right) \in \check{h}_{1}$, and $a_{j}=\left(s_{\theta(j)}, \operatorname{lh}\left(s_{\theta(j)}\right)\right) \in \breve{h}_{2}$. Some operation laws can be defined as follows:

$$
\begin{gathered}
(1) \check{h}_{1} \oplus \check{h}_{2}=\underset{a_{a_{i}} \check{\epsilon}_{1}, a_{j} \check{h}_{2}}{=}\left\{\left(s_{\theta(i)+\theta(j)},\right.\right. \\
\left.\left.\bigcup_{\left(\mu_{i}, v_{i}\right) \in \ln \left(s_{\theta(i)}\right),\left(\mu_{j}, v_{j}\right) \in l h\left(s_{\theta(j)}\right)}\left\{\left(\mu_{i}+\mu_{j}-\mu_{i} \mu_{j}, v_{i} \nu_{j}\right)\right\}\right)\right\},
\end{gathered}
$$

$(2) \check{h}_{1} \otimes \check{h}_{2} \quad=\quad \bigcup_{a_{i} \check{h}_{1}, a_{j} \in \check{h}_{2}}\left\{\left(s_{\theta(i) \theta(j)}\right.\right.$, $\left.\left.\bigcup_{\left(\mu_{i}, v_{i}\right) \in \ln \left(s_{\theta(i)}\right),\left(\mu_{j}, v_{j}\right) \in \operatorname{ll}\left(s_{\theta(j)}\right)}\left\{\left(\mu_{i} \mu_{j}, \nu_{i}+v_{j}-v_{i} \nu_{j}\right)\right\}\right)\right\}$,

(3) $\lambda \check{h}=\bigcup_{a_{k} \in \check{h}}\left\{\left(s_{\lambda \theta(k)}, \bigcup_{\left(\mu_{k}, v_{k}\right) \in \operatorname{lh}\left(s_{\theta(k)}\right)}\left\{\left(1-\left(1-\mu_{k}\right)^{\lambda}, v_{k}^{\lambda}\right)\right\}\right)\right\}$,

(4) $(\check{h})^{\lambda}=\bigcup_{a_{k} \in \check{h}}\left\{\left(s_{\theta(k)^{\lambda}}, \bigcup_{\left(\mu_{k}, \nu_{k}\right) \in \operatorname{lh}\left(s_{\theta(k)}\right)}\left\{\left(\mu_{k}^{\lambda}, 1-(1-\right.\right.\right.\right.$ $\left.\left.\left.\left.\left.v_{k}\right)^{\lambda}\right)\right\}\right)\right\}$.

Definition 5. Let $a_{i}=\left(s_{\theta(i)}, \operatorname{lh}\left(s_{\theta(i)}\right)\right)$ be a LIFE, then the score function $s\left(a_{i}\right)$ of $a_{i}$ can be defined as

$$
s\left(a_{i}\right)=\frac{\theta(i)}{g\left|\operatorname{lh}\left(s_{\theta(i)}\right)\right|} \sum_{\left(\alpha_{i}, \beta_{i}\right) \in \ln \left(s_{\theta(i)}\right)}\left(\alpha_{i}-\beta_{i}\right),
$$

and the accuracy function $h\left(a_{i}\right)$ of $a_{i}$ can be defined as

$$
h\left(a_{i}\right)=\frac{\theta(i)}{g\left|l h\left(s_{\theta(i)}\right)\right|} \sum_{\left(\alpha_{i}, \beta_{i}\right) \in \ln \left(s_{\theta(i)}\right)}\left(\alpha_{i}+\beta_{i}\right),
$$

where $g$ is the number of linguistic arguments in linguistic term set $S$ and $\left|l h\left(s_{\theta(i)}\right)\right|$ is the number of intuitionistic fuzzy memberships in $\operatorname{lh}\left(s_{\theta(i)}\right)$.
Let $a_{i}=\left(s_{\theta(i)}, \operatorname{lh}\left(s_{\theta(i)}\right)\right)$ and $a_{j}=\left(s_{\theta(j)}, \operatorname{lh}\left(s_{\theta(j)}\right)\right)$ be two LIFEs. Then, $a_{i}$ and $a_{j}$ can be ranked as follows:

(1) if $s\left(a_{i}\right)<s\left(a_{j}\right)$, then $a_{i}<a_{j}$,

(2) if $s\left(a_{i}\right)=s\left(a_{j}\right)$ and

(I) if $h\left(a_{i}\right)<h\left(a_{j}\right)$, then $a_{i}<a_{j}$,

(II) if $h\left(a_{i}\right)=h\left(a_{j}\right)$, then $a_{i}=a_{j}$.

Definition 6. Let $\check{h}=\left\{\left(s_{\theta(i)}, \operatorname{lh}\left(s_{\theta(i)}\right)\right) \mid\left(s_{\theta(i)}, \operatorname{lh}\left(s_{\theta(i)}\right)\right) \in \check{h}\right\}$ be LHIFE; the score function $S(\check{h})$ can be defined as

$$
S(\check{h})=\frac{1}{l_{\grave{h}}}\left(\sum_{i=1}^{l_{\grave{h}}} \frac{\theta(i)}{g\left|\operatorname{lh}\left(s_{\theta(i)}\right)\right|} \sum_{\left(\alpha_{i}, \beta_{i}\right) \in \operatorname{lh}\left(s_{\theta(i)}\right)}\left(\alpha_{i}-\beta_{i}\right)\right) .
$$

The accuracy function $A(\check{h})$ can be defined as

$$
A(\check{h})=\frac{1}{l_{\check{h}}}\left(\sum_{i=1}^{l_{\grave{h}}} \frac{\theta(i)}{g\left|\operatorname{lh}\left(s_{\theta(i)}\right)\right|} \sum_{\left(\alpha_{i}, \beta_{i}\right) \in \operatorname{lh}\left(s_{\theta(i)}\right)}\left(\alpha_{i}+\beta_{i}\right)\right) .
$$

Here, $l_{\check{h}}$ is the number of LIFEs in $\check{h}$ and $g$ is the number of linguistic arguments in linguistic term set $S$, and $\left|h h\left(s_{\theta(i)}\right)\right|$ is the count of intuitionistic fuzzy memberships in $\operatorname{lh}\left(s_{\theta(i)}\right)$.

Based on the score function and accuracy function, we present the following method to compare LHIFEs. Let $\check{h}_{1}$ and $\check{h}_{2}$ be two LHIFEs as follows:

(1) if $S\left(\check{h}_{1}\right)<S\left(\check{h}_{2}\right)$, then $\check{h}_{1}<\check{h}_{2}$;

(2) if $S\left(\check{h}_{1}\right)=S\left(\check{h}_{2}\right)$ and

$$
\begin{aligned}
& \text { (I) } A\left(\check{h}_{1}\right)<A\left(\check{h}_{2}\right) \text {, then } \check{h}_{1}<\check{h}_{2}, \\
& \text { (II) } A\left(\check{h}_{1}\right)=A\left(\check{h}_{2}\right) \text {, then } \check{h}_{1}=\check{h}_{2} .
\end{aligned}
$$

\section{Aggregation Operators for Linguistic Hesitant Intuitionistic Fuzzy Information}

In this section, we define some hybrid aggregation operators based on the linguistic hesitant intuitionistic fuzzy information.

Definition 7. Let $X=\left\{x_{1}, x_{2}, \ldots, x_{n}\right\}$ be a reference set and let $\check{h}_{j}(j=1,2, \ldots, n)$ be a collection of LHIFEs on $X$. A generalized linguistic hesitant intuitionistic fuzzy hybrid weighted averaging (GLHIFHWA) operator is a mapping GLHIFHWA: $H^{n} \rightarrow H$, which can be defined as follows:

$$
\begin{aligned}
& \operatorname{GLHIFHWA}\left(\check{h}_{1}, \check{h}_{2}, \ldots, \check{h}_{n}\right) \\
& =\left(\frac{\bigoplus_{j=1}^{n} w_{j} \omega_{\check{h}_{\sigma(j)}}^{\lambda} \check{h}_{\sigma(j)}^{\lambda}}{\sum_{j=1}^{n} w_{j} \omega_{\check{h}_{\sigma(j)}}^{\lambda}}\right)^{1 / \lambda},
\end{aligned}
$$

where $\lambda \in(-\infty,+\infty)$ and $w=\left(w_{1}, w_{2}, \ldots, w_{n}\right)$ is the weight vector on the ordered set $N=\{1,2, \ldots, n\}$ with $w_{i} \geq 0$ and 
$\sum_{j=1}^{n} w_{j}=1 . \omega=\left(\omega_{\check{h}_{1}}, \omega_{\check{h}_{2}}, \ldots, \omega_{\breve{h}_{n}}\right)$ is the weight vector of LHIFEs $\check{h}_{j}(j=1,2, \ldots, n)$ such that $\omega_{\check{h}_{j}} \geq 0$ and $\sum_{j=1}^{n} \omega_{\check{h}_{j}}=$ 1. $(\sigma(1), \sigma(2), \ldots, \sigma(n))$ is a permutation of $(1,2, \ldots, n)$ such that $\omega_{\check{h}_{\sigma(j)}} \check{h}_{\sigma(j)}$ is the $j$ th largest $\omega_{\check{h}_{j}} \check{h}_{j}$.

Definition 8. Let $X=\left\{x_{1}, x_{2}, \ldots, x_{n}\right\}$ be a reference set and let $\check{h}_{j}(j=1,2, \ldots, n)$ be a collection of LHIFEs on $X$. A generalized linguistic hesitant intuitionistic fuzzy hybrid geometric mean (GLHIFHGM) operator is a mapping GLHIFHGM: $H^{n} \rightarrow H$, which can be defined as follows:

$$
\begin{aligned}
& \operatorname{GLHIFHGM}\left(\check{h}_{1}, \check{h}_{2}, \ldots, \check{h}_{n}\right) \\
& =\frac{1}{\lambda}\left(\bigotimes_{j=1}^{n}\left(\lambda \check{h}_{\sigma(j)}\right)^{w_{j} \omega_{h_{\sigma(j)}}^{\lambda} / \sum_{j=1}^{n} w_{j} \omega_{h_{\sigma(j)}}^{\lambda}}\right),
\end{aligned}
$$

where $\lambda \in(-\infty,+\infty)$ and $w=\left(w_{1}, w_{2}, \ldots, w_{n}\right)$ is the weight vector on the ordered set $N=\{1,2, \ldots, n\}$ with $w_{i} \geq 0$ and $\sum_{j=1}^{n} w_{j}=1 . \omega=\left(\omega_{\check{h}_{1}}, \omega_{\check{h}_{2}}, \ldots, \omega_{\check{h}_{n}}\right)$ is the weight vector of LHIFEs $\check{h}_{j}(j=1,2, \ldots, n)$ such that $\omega_{\check{h}_{j}} \geq 0$ and $\sum_{j=1}^{n} \omega_{\check{h}_{j}}=$ 1. $(\sigma(1), \sigma(2), \ldots, \sigma(n))$ is a permutation of $(1,2, \ldots, n)$ such that $\check{h}_{\sigma(j)}^{\omega_{\sigma(j)}}$ is the $j$ th largest $\check{h}_{j}^{\omega_{j}}$.

Let $\check{h}_{j}(j=1,2, \ldots, n)$ be a collection of LHIFEs and $a_{i}=\left(s_{\theta(i)}, l h\left(s_{\theta(i)}\right)\right) \in \check{h}_{i}$. By using the operation laws of LHIFEs, we can get the values of the GLHIFHWA operator and the GLHIFHGM operator, which are all LHIFEs and

$$
\begin{aligned}
& \operatorname{GLHIFHWA}\left(\check{h}_{1}, \check{h}_{2}, \ldots, \check{h}_{n}\right)=\bigcup_{a_{\sigma(i)} \check{h}_{\sigma(i)}}\left\{\left(s_{\left(\sum_{j=1}^{n}\left(w_{j}\left(\omega_{\check{h}_{\sigma(j)}} \theta(\sigma(j))\right)^{\lambda} / \sum_{j=1}^{n} w_{j} \omega_{h_{\sigma(j)}}^{\lambda}\right)\right)^{1 / \lambda}}\right.\right. \\
& \left.\left.\bigcup_{\left(\mu_{\sigma(i)}, v_{\sigma(i)}\right) \in \operatorname{ll}\left(s_{\theta_{\sigma(i)}}\right)}\left\{\left(\left(1-\prod_{j=1}^{n}\left(1-\mu_{\sigma(j)}^{\lambda}\right)^{w_{j} \omega_{h_{\sigma(j)}}^{\lambda} / \sum_{j=1}^{n} w_{j} \omega_{h_{\sigma(j)}}^{\lambda}}\right)^{1 / \lambda}, 1-\left(1-\prod_{j=1}^{n}\left(1-\left(1-v_{\sigma(j)}\right)^{\lambda}\right)^{w_{j} \omega_{h_{\sigma(j)}}^{\lambda} / \sum_{j=1}^{n} w_{j} \omega_{h_{\sigma(j)}}^{\lambda}}\right)^{1 / \lambda}\right)\right\}\right)\right\} \text {, } \\
& \operatorname{GLHIFHGM}\left(\check{h}_{1}, \check{h}_{2}, \ldots, \check{h}_{n}\right)=\bigcup_{a_{\sigma(i)} \in \check{h}_{\sigma(i)}}\left\{\left(s \prod_{j=1}^{n}(\sigma(j)){ }^{\left.w_{j} \omega_{h_{\sigma(j)}} / \Sigma_{j=1}^{n} w_{j} \omega_{h_{\sigma(j)}}\right)},\right.\right. \\
& \left.\left.\bigcup_{\left(\mu_{\sigma(i)}, v_{\sigma(i)}\right) \in l\left(s_{\theta_{\sigma(i)}}\right)}\left\{\left(1-\left(1-\prod_{j=1}^{n}\left(1-\left(1-\mu_{\sigma(j)}\right)^{\lambda}\right)^{w_{j} \omega_{\check{h}_{\sigma(j)}} / \sum_{j=1}^{n} \omega_{j} \omega_{\bar{h}_{\sigma(j)}}}\right)^{1 / \lambda},\left(1-\prod_{j=1}^{n}\left(1-v_{\sigma(j)}^{\lambda}\right)^{w_{j} \omega_{\bar{h}_{\sigma(j)}} / \sum_{j=1}^{n} w_{j} \omega_{\bar{h}_{\sigma(j)}}}\right)^{1 / \lambda}\right)\right\}\right)\right\} .
\end{aligned}
$$

Now, we consider some special cases of the GLHIFHWA operator and the GLHIFHGM operator by considering some special $\lambda$ and the associated weight vectors $w=$ $\left(w_{1}, w_{2}, \ldots, w_{n}\right)$ and $\omega=\left(\omega_{1}, \omega_{2}, \ldots, \omega_{n}\right)$.

Remark 9. If all $\left(\mu_{i}, \nu_{i}\right) \in \operatorname{lh}\left(s_{\theta(i)}\right)$ reduces to $\left(\mu_{i}, 1-\mu_{i}\right)$, then LHIFEs $\check{h}_{j}(j=1,2, \ldots, n)$ become linguistic hesitant fuzzy elements (LHFEs) $h_{j}^{\prime}(j=1,2, \ldots, n)$ and GLHIFHWA operator becomes the following generalized linguistic hesitant fuzzy hybrid weighted averaging (GLHFHWA) operator:

$$
\operatorname{GLHFHWA}\left(h_{1}^{\prime}, h_{2}^{\prime}, \ldots, h_{n}^{\prime}\right)=\left(\frac{\bigoplus_{j=1}^{n} w_{j}\left(\omega_{h_{\sigma(j)}^{\prime}} h_{\sigma(j)}^{\prime}\right)^{\lambda}}{\sum_{j=1}^{n} w_{j} \omega_{h_{\sigma(j)}^{\prime}}^{\lambda}}\right)^{1 / \lambda}
$$

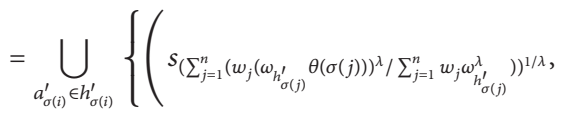

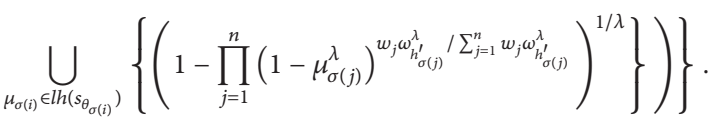

The GLHIFHGM operator becomes the generalized linguistic hesitant fuzzy hybrid geometric mean (GLHFHGM) operator as follows:

$$
\begin{aligned}
& \operatorname{GLHFHGM}\left(h_{1}^{\prime}, h_{2}^{\prime}, \ldots, h_{n}^{\prime}\right)=\frac{1}{\lambda}\left(\bigotimes_{j=1}^{n}\left(\lambda h_{\sigma(j)}^{\prime}\right)^{w_{j} \omega_{h_{\sigma(j)}^{\prime}} / \sum_{j=1}^{n} w_{j} \omega_{h_{\sigma(j)}^{\prime}}}\right) \\
& =\bigcup_{a_{\sigma(i)}^{\prime} \in h_{\sigma(i)}^{\prime}}\left\{\left(s_{\prod_{j=1}^{n} \theta(\sigma(j))}^{w_{j} \omega_{h_{(j)}^{\prime}}\left(\sum_{j=1}^{n} w_{j} \omega_{h_{\sigma(j)}^{\prime}},\right.} \bigcup_{\mu_{\sigma(i)} \in l h\left(s_{\theta_{\sigma(i)}}\right)}\left\{1-\left(1-\prod_{j=1}^{n}\left(1-\left(1-\mu_{\sigma(j)}\right)\right)^{w_{j} \omega_{h_{\sigma(j)}^{\prime}} / \sum_{j=1}^{n} w_{j} \omega_{\left.h_{\sigma(j)}^{\prime}\right)}}\right)^{1 / \lambda}\right\}\right)\right\} .
\end{aligned}
$$


If all $\left(\mu_{i}, v_{i}\right) \in \operatorname{lh}\left(s_{\theta(i)}\right)$ reduces to $(1,0)$, then LHIFEs $\check{h}_{i}(i=1,2, \ldots, n)$ reduce to linguistic hesitant elements $h_{i}=$ $\left\{s_{\theta(i)}\right\}(i=1,2, \ldots, n)$. The GLHIFHWA operator becomes the generalized linguistic hesitant hybrid weighted averaging (GLHHWA) operator as follows:

$$
\begin{aligned}
& \operatorname{GLHhWA}\left(h_{1}, h_{2}, \ldots, h_{n}\right) \\
& =\left(\frac{\bigoplus_{j=1}^{n} w_{j}\left(\omega_{h_{\sigma(j)}} h_{\sigma(j)}\right)^{\lambda}}{\sum_{j=1}^{n} w_{j} \omega_{h_{\sigma(j)}}^{\lambda}}\right)^{1 / \lambda} \\
& =\bigcup_{s_{\theta(\sigma(j))} \in h_{\sigma(i)}}\left\{s_{\left.\left(\sum_{j=1}^{n}\left(w_{j}\left(\omega_{h_{\sigma(j)}} \theta(\sigma(j))\right)^{\lambda} / \sum_{j=1}^{n} w_{j} \omega_{h_{\sigma(j)}}^{\lambda}\right)\right)^{1 / \lambda}\right\} .}\right.
\end{aligned}
$$

The GLHIFHGM operator becomes the generalized linguistic hesitant hybrid geometric mean (GLHHGM) operator as follows:

$$
\begin{aligned}
& \operatorname{GLHHGM}\left(h_{1}, h_{2}, \ldots, h_{n}\right) \\
& =\frac{1}{\lambda}\left(\bigotimes_{j=1}^{n}\left(\lambda h_{\sigma(j)}\right)^{w_{j} \omega_{h_{\sigma(j)}} / \sum_{j=1}^{n} w_{j} \omega_{h_{\sigma(j)}}}\right)
\end{aligned}
$$

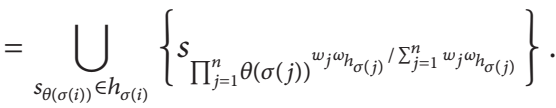

Remark 10. If $\lambda=1$, then the GLHIFHWA operator becomes the linguistic hesitant intuitionistic fuzzy hybrid weighted averaging (LHIFHWA) operator

$$
\begin{array}{r}
\operatorname{LHIFHWA}\left(\check{h}_{1}, \check{h}_{2}, \ldots, \check{h}_{n}\right)=\frac{\bigoplus_{j=1}^{n} w_{j} \omega_{\check{h}_{\sigma(j)}} \check{h}_{\sigma(j)}}{\sum_{j=1}^{n} w_{j} \omega_{\check{h}_{\sigma(j)}}}=\bigcup_{a_{\sigma(i)} \in \check{h}_{\sigma(i)}}\left\{\left(s_{\left(\sum_{j=1}^{n} w_{j} \omega_{\check{h}_{\sigma(j)}} \theta(\sigma(j)) / \sum_{j=1}^{n} w_{j} \omega_{h_{\sigma(j)}}\right.},\right.\right. \\
\left.\left.\bigcup_{\left(\mu_{\sigma(i)}, v_{\sigma(i)}\right) \in l h\left(s_{\theta_{\sigma(i)}}\right)}\left\{\left(1-\prod_{j=1}^{n}\left(1-\mu_{\sigma(j)}\right)^{w_{j} \omega_{\tilde{h}_{\sigma(j)}} / \sum_{j=1}^{n} w_{j} \omega_{\check{h}_{\sigma(j)}}}, \prod_{j=1}^{n}\left(v_{\sigma(j)}\right)^{w_{j} \omega_{\check{h}_{\sigma(j)}} / \sum_{j=1}^{n} w_{j} \omega_{\check{h}_{\sigma(j)}}}\right)\right\}\right)\right\},
\end{array}
$$

and the GLHIFHGM operator becomes the linguistic hesitant intuitionistic fuzzy hybrid geometric mean (LHIFHGM) operator

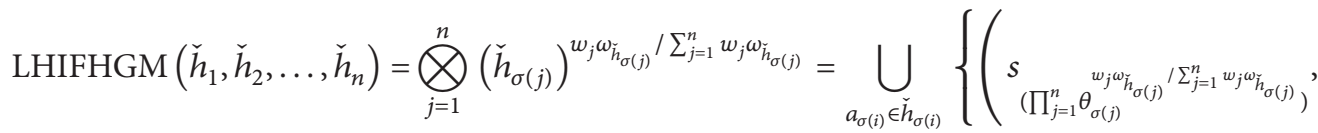

$$
\begin{aligned}
& \left.\left.\bigcup_{\left(\mu_{\sigma(i)}, v_{\sigma(i)}\right) \in l h\left(s_{\sigma_{\sigma(i)}}\right)}\left\{\left(\prod_{j=1}^{n}\left(v_{\sigma(j)}\right)^{w_{j} \omega_{\tilde{h}_{\sigma(j)}} / \sum_{j=1}^{n} w_{j} \omega_{h_{\sigma(j)}}}, 1-\prod_{j=1}^{n}\left(1-\mu_{\sigma(j)}\right)^{w_{j} \omega_{h_{\sigma(j)}} / \sum_{j=1}^{n} w_{j} \omega_{h_{\sigma(j)}}}\right)\right\}\right)\right\} .
\end{aligned}
$$

Remark 11. If $\lambda=2$, then the GLHIFHWA operator reduces to be the linguistic hesitant intuitionistic fuzzy hybrid weighted quadratic averaging (LHIFHWQA) operator as follows:

$$
\begin{aligned}
& \operatorname{LHIFHWQA}\left(\check{h}_{1}, \check{h}_{2}, \ldots, \check{h}_{n}\right)=\bigcup_{a_{\sigma(i)} \check{h}_{\sigma(i)}}\left\{\left(s_{\left(\sum_{j=1}^{n}\left(w_{j}\left(\omega_{h_{\sigma(j)}} \theta(\sigma(j))\right)^{2} / \sum_{j=1}^{n} w_{j} \omega_{h_{\sigma(j)}}^{2}\right)\right)^{1 / 2},}\right.\right. \\
& \left.\left.\bigcup_{\left(\mu_{\sigma(i)}, v_{\sigma(i)}\right) \in \operatorname{li}\left(s_{\theta_{\sigma(i)}}\right)}\left\{\left(\left(1-\prod_{j=1}^{n}\left(1-\mu_{\sigma(j)}^{2}\right)^{w_{j} \omega_{\omega_{\sigma(j)}}^{2} / \sum_{j=1}^{n} w_{j} \omega_{\omega_{\sigma(j)}}^{2}}\right)^{1 / 2}, 1-\left(1-\prod_{j=1}^{n}\left(1-\left(1-v_{\sigma(j)}\right)^{2}\right)^{w_{j} \omega_{h_{\sigma(j)}}^{2} / \sum_{j=1}^{n} w_{j} \omega_{h_{\sigma(j)}}^{2}}\right)^{1 / 2}\right)\right\}\right)\right\} .
\end{aligned}
$$


The GLHIFHWA operator becomes the linguistic hesitant intuitionistic fuzzy hybrid quadratic geometric mean (LHIFHQGM) operator

$$
\begin{aligned}
& \operatorname{LHIFHQGM}\left(\check{h}_{1}, \check{h}_{2}, \ldots, \check{h}_{n}\right)=\bigcup_{a_{\sigma(i)} \in \check{h}_{\sigma(i)}}\left\{\left(s_{\prod_{j=1}^{n} \theta(\sigma(j))}^{w_{j} \omega_{\check{h}_{\sigma(j)}} / \sum_{j=1}^{n} w_{j} \omega_{\tilde{h}_{\sigma(j)}},}\right.\right. \\
& \left.\qquad \bigcup_{\left(\mu_{\sigma(i)}, v_{\sigma(i)}\right) \in \operatorname{lh}\left(s_{\theta_{\sigma(i)}}\right)}\left\{\left(1-\left(1-\prod_{j=1}^{n}\left(1-\left(1-\mu_{\sigma(j)}\right)^{2}\right)^{w_{j} \omega_{\check{h}_{\sigma(j)}} / \sum_{j=1}^{n} w_{j} \omega_{\check{h}_{\sigma(j)}}}\right)^{1 / 2},\left(1-\prod_{j=1}^{n}\left(1-v_{\sigma(j)}^{2}\right)^{\left.w_{j} \omega_{\check{h}_{\sigma(j)}} / \sum_{j=1}^{n} w_{j} \omega_{\check{h}_{\sigma(j)}}\right)}\right)\right\}\right)\right\} .
\end{aligned}
$$

If all $\left(\mu_{i}, v_{i}\right) \in \operatorname{lh}\left(s_{\theta(i)}\right)$ reduces to $\left(\mu_{i}, 1-\mu_{i}\right)$, then LHIFHWQA operator becomes the linguistic hesitant fuzzy hybrid weighted quadratic averaging (LHFHWQA) operator as follows:

$$
\begin{aligned}
& \operatorname{LHFHWQA}\left(h_{1}^{\prime}, h_{2}^{\prime}, \ldots, h_{n}^{\prime}\right) \\
& =\bigcup_{a_{\sigma(i)}^{\prime} \in h_{\sigma(i)}^{\prime}}\left\{\left(s_{\left(\sum_{j=1}^{n}\left(w_{j} \omega_{\sigma(j)}^{2} / \sum_{j=1}^{n} w_{j} \omega_{h_{\sigma(j)}^{2}}^{2}\right)(\theta(\sigma(j)))^{2}\right)^{1 / 2},}\right.\right.
\end{aligned}
$$

$$
\left.\left.\bigcup_{\mu_{\sigma(i)} \in \operatorname{lh}\left(s_{\theta_{\sigma(i)}}\right)}\left\{\left(1-\prod_{j=1}^{n}\left(1-\mu_{\sigma(j)}^{2}\right)^{w_{j} \omega_{h_{\sigma(j)}^{2}}^{2} / \sum_{j=1}^{n} w_{j} \omega_{h_{\sigma(j)}}^{2}}\right)^{1 / 2}\right\}\right)\right\}
$$

The LHIFHQGM operator becomes the linguistic hesitant fuzzy hybrid quadratic geometric mean (LHIFHQGM) operator

$$
\begin{aligned}
& \operatorname{LHIFHQGM}\left(h_{1}^{\prime}, h_{2}^{\prime}, \ldots, h_{n}^{\prime}\right)=\bigcup_{a_{\sigma(i)} \in h_{\sigma(i)}^{\prime}}\left\{\left(\begin{array}{l}
s_{\prod_{j=1}^{n} \theta(\sigma(j))} w_{j \omega_{h_{\sigma(j)}^{\prime}}} / \sum_{j=1}^{n} w_{j} \omega_{h_{\sigma(j)}^{\prime}},
\end{array}\right.\right. \\
& \left.\left.\bigcup_{\left(\mu_{\sigma(i)}, v_{\sigma(i)}\right) \in \operatorname{lh}\left(s_{\theta_{\sigma(i)}}\right)}\left\{1-\left(1-\prod_{j=1}^{n}\left(1-\left(1-\mu_{\sigma(j)}\right)^{2}\right)^{w_{j} \omega_{h_{\sigma(j)}^{\prime}} / \sum_{j=1}^{n} w_{j} \omega_{h_{\sigma(j)}^{\prime}}}\right)^{1 / 2}\right\}\right)\right\} .
\end{aligned}
$$

If all $\left(\mu_{i}, v_{i}\right) \in \operatorname{lh}\left(s_{\theta(i)}\right)$ reduces to $(1,0)$, then LHIFHWQA operator becomes the linguistic hesitant hybrid weighted quadratic averaging (LHHWQA) operator as follows:

$$
\begin{aligned}
& \operatorname{LHHWQA}\left(h_{1}, h_{2}, \ldots, h_{n}\right) \\
& =\bigcup_{s_{\theta(\sigma(i))} \in h_{\sigma(i)}}\left\{s_{\left.\left(\sum_{j=1}^{n}\left(w_{j}\left(\omega_{h_{\sigma(j)}} \theta(\sigma(j))\right)^{2} / \sum_{j=1}^{n} w_{j} \omega_{h_{\sigma(j)}^{2}}^{2}\right)\right)^{1 / 2}\right\} .}\right.
\end{aligned}
$$

The LHIFHQGM operator becomes the linguistic hesitant hybrid weighted geometric mean (LHHWGM) operator

$$
\begin{aligned}
& \operatorname{LHHWGM}\left(h_{1}, h_{2}, \ldots, h_{n}\right) \\
& =\bigcup_{s_{\theta(\sigma(i))} \in h_{\sigma(i)}}\left\{s_{\prod_{j=1}^{n} \theta(\sigma(j))}^{w_{j} \omega_{h_{\sigma(j)}} / \sum_{j=1}^{n} w_{j} \omega_{h_{\sigma(j)}}}\right\} .
\end{aligned}
$$

Remark 12. If $w_{j}=1 / n(j=1,2, \ldots, n)$, the GLHIFHWA operator degenerates to the following generalized linguistic hesitant intuitionistic fuzzy weighted averaging (GLHIFWA) operator:

$$
\begin{aligned}
& \operatorname{GLHIFWA}\left(\check{h}_{1}, \check{h}_{2}, \ldots, \check{h}_{n}\right)=\bigcup_{a_{i} \in \check{h}_{i}}\left\{\left(s_{\left(\sum_{j=1}^{n}\left(\omega_{\check{h}_{j}}^{\lambda} / \sum_{j=1}^{n} \omega_{\check{h}_{j}}^{\lambda}\right)(\theta(j))^{\lambda}\right)^{1 / \lambda}}\right.\right. \\
& \left.\left.\bigcup_{\left(\mu_{i}, \nu_{i}\right) \in \operatorname{lh}\left(s_{\theta_{j}}\right)}\left\{\left(\left(1-\prod_{j=1}^{n}\left(1-\mu_{j}^{\lambda}\right)^{\omega_{h_{j}}^{\lambda} / \sum_{j=1}^{n} \omega_{h_{j}}^{\lambda}}\right)^{1 / \lambda}, 1-\left(1-\prod_{j=1}^{n}\left(1-\left(1-v_{j}\right)^{\lambda}\right)^{\omega_{h_{j}}^{\lambda} / \sum_{j=1}^{n} \omega_{\hat{h}_{j}}^{\lambda}}\right)^{1 / \lambda}\right)\right\}\right)\right\} .
\end{aligned}
$$


The GLHIFHGM operator becomes the generalized linguistic hesitant intuitionistic fuzzy weighted geometric mean (GLHIFWGM) operator:

$$
\begin{aligned}
& \operatorname{GLHIFWGM}\left(\check{h}_{1}, \check{h}_{2}, \ldots, \check{h}_{n}\right) \\
& \quad=\bigcup_{a_{i} \in \check{h}_{i}}\left\{\left(s_{\prod_{j=1}^{n} \theta(j)}^{\omega_{\check{h}}}, \bigcup_{\left(\mu_{i}, v_{i}\right) \in l h\left(s_{\theta_{i}}\right)}\left\{\left(1-\left(1-\prod_{j=1}^{n}\left(1-\left(1-\mu_{j}\right)^{\lambda}\right)^{\omega_{\check{h}_{j}}}\right)^{1 / \lambda},\left(1-\prod_{j=1}^{n}\left(1-v_{j}^{\lambda}\right)^{\omega_{\check{h}_{j}}}\right)\right)^{1 / \lambda}\right)\right\} .\right.
\end{aligned}
$$

If $\lambda=1$ and $w_{j}=1 / n$ for all $j=1,2, \ldots, n$, the GLHIFHWA operator becomes the linguistic hesitant intuitionistic fuzzy weighted averaging (LHIFWA) operator:

$$
\begin{aligned}
& \operatorname{LHIFWA}\left(\check{h}_{1}, \check{h}_{2}, \ldots, \check{h}_{n}\right)=\bigoplus_{j=1}^{n} \omega_{\check{h}_{j}} \check{h}_{j} \\
& =\bigcup_{a_{i} \in \check{h}_{i}}\left\{\left(s_{\sum_{j=1}^{n} \omega_{\check{h}_{j}} \theta(j)}\right.\right. \\
& \left.\left.\bigcup_{\left(\mu_{i}, \nu_{i}\right) \in \operatorname{lh}\left(s_{\theta_{i}}\right)}\left\{\left(1-\prod_{j=1}^{n}\left(1-\mu_{j}\right)^{\omega_{\check{h}_{j}}}, \prod_{j=1}^{n} v_{j}^{\omega_{\check{h}_{j}}}\right)\right\}\right)\right\} .
\end{aligned}
$$

The GLHIFHGM operator becomes the linguistic hesitant intuitionistic fuzzy weighted geometric mean (LHIFWGM) operator

$$
\begin{gathered}
\operatorname{LHIFWGM}\left(\check{h}_{1}, \check{h}_{2}, \ldots, \check{h}_{n}\right)=\bigcup_{a_{i} \in \check{h}_{i}}\left\{\left(s_{\prod_{j=1}^{n}(\theta(j))}^{\omega_{\check{h}_{j}},}\right.\right. \\
\left.\left.\qquad \bigcup_{\left(\mu_{i}, v_{i}\right) \in \operatorname{lh}\left(s_{\theta_{i}}\right)}\left\{\left(\prod_{j=1}^{n} \mu_{j}^{\omega_{\check{h}_{j}}}, 1-\prod_{j=1}^{n}\left(1-v_{j}\right)^{\omega_{\check{h}_{j}}}\right)\right\}\right)\right\} .
\end{gathered}
$$

Remark 13. If $\omega_{\check{h}_{j}}=1 / n(j=1,2, \ldots, n)$, the GLHIFHWA operator degenerates to the generalized linguistic hesitant intuitionistic fuzzy ordered weighted averaging (GLHIFOWA) operator:

$$
\begin{aligned}
& \operatorname{GLHIFOWA}\left(\check{h}_{1}, \check{h}_{2}, \ldots, \check{h}_{n}\right)=\left(\bigoplus_{j=1}^{n} w_{j} \check{h}_{\sigma(j)}^{\lambda}\right)^{1 / \lambda} \\
& =\bigcup_{a_{\sigma(i)} \in \check{h}_{\sigma(i)}}\left\{\left(s_{\left(\sum_{j=1}^{n} w_{j}(\theta(\sigma(j)))^{\lambda}\right)^{1 / \lambda}}\right.\right. \\
& \left.\left.\bigcup_{\left(\mu_{\sigma(i)}, v_{\sigma(i)}\right) \in \operatorname{lh}\left(s_{\theta_{\sigma(i)}}\right)}\left\{\left(\left(1-\prod_{j=1}^{n}\left(1-\mu_{\sigma(j)}^{\lambda}\right)^{w_{j}}\right)^{1 / \lambda}, 1-\left(1-\prod_{j=1}^{n}\left(1-\left(1-v_{\sigma(j)}\right)^{\lambda}\right)^{w_{j}}\right)^{1 / \lambda}\right)\right\}\right)\right\} .
\end{aligned}
$$

The GLHIFHGM operator becomes the generalized linguistic hesitant intuitionistic fuzzy ordered geometric mean (GLHIFOGM) operator:

$$
\begin{aligned}
& \operatorname{GLhifogm}\left(\check{h}_{1}, \check{h}_{2}, \ldots, \check{h}_{n}\right)=\bigcup_{a_{\sigma(i)} \check{h}_{\sigma(i)}}\left\{\left(s_{\prod_{j=1}^{n}(\theta(\sigma(j)))^{w_{j}}}\right.\right. \\
& \left.\left.\qquad \bigcup_{\left(\mu_{\sigma(i)}, v_{\sigma(i)}\right) \in \operatorname{lh}\left(s_{\theta_{\sigma(i)}}\right)}\left\{\left(1-\left(1-\prod_{j=1}^{n}\left(1-\left(1-\mu_{\sigma(j)}\right)^{\lambda}\right)^{w_{j}}\right)^{1 / \lambda},\left(1-\prod_{j=1}^{n}\left(1-v_{\sigma(j)}^{\lambda}\right)^{w_{j}}\right)^{1 / \lambda}\right)\right\}\right)\right\} .
\end{aligned}
$$


If $\lambda=1$ and $\omega_{\check{h}_{j}}=1 / n(j=1,2, \ldots, n)$, the GLHIFHWA operator becomes the linguistic hesitant intuitionistic fuzzy ordered weighted averaging (LHIFOWA) operator as follows:

$$
\begin{aligned}
& \operatorname{LHIFOWA}\left(\check{h}_{1}, \check{h}_{2}, \ldots, \check{h}_{n}\right)=\bigoplus_{j=1}^{n} w_{j} \check{h}_{\sigma(j)} \\
& =\bigcup_{a_{\sigma(i)} \in \check{h}_{\sigma(i)}}\left\{\left(s_{\sum_{j=1}^{n} w_{j} \theta(\sigma(j)),}\right.\right. \\
& \left.\left.\bigcup_{\left(\mu_{\sigma(i)}, v_{\sigma(i)}\right) \in \operatorname{ll}\left(s_{\theta_{\sigma(i)}}\right)}\left\{\left(1-\prod_{j=1}^{n}\left(1-\mu_{\sigma(j)}\right)^{w_{j}}, \prod_{j=1}^{n} v_{\sigma(j)}^{w_{j}}\right)\right\}\right)\right\} .
\end{aligned}
$$

The GLHIFHGM operator becomes the linguistic hesitant intuitionistic fuzzy ordered weighted geometric mean (LHIFOWGM) operator:

$$
\begin{aligned}
& \operatorname{LHIFOWGM}\left(\check{h}_{1}, \check{h}_{2}, \ldots, \check{h}_{n}\right)=\bigcup_{a_{\sigma(i)} \check{h}_{\sigma(i)}}\left\{\left(s_{\prod_{j=1}^{n} \theta(\sigma(j))^{w_{j}}},\right.\right. \\
& \left.\left.\bigcup_{\left(\mu_{\sigma(i)}, v_{\sigma(i)}\right) \in \operatorname{lh}\left(s_{\theta_{\sigma(i)}}\right)}\left\{\left(\prod_{j=1}^{n} \mu_{\sigma(j)}^{w_{j}}, 1-\prod_{j=1}^{n}\left(1-v_{\sigma(j)}\right)^{w_{j}}\right)\right\}\right)\right\} .
\end{aligned}
$$

If $\lambda=0$ and $\omega_{\breve{h}_{j}}=1 / n(j=1,2, \ldots, n)$, the GLHIFHWA operator becomes the following linguistic hesitant intuitionistic fuzzy ordered weighted aggregation operator:

$$
\begin{aligned}
& \operatorname{GLHIFHWA}\left(\check{h}_{1}, \check{h}_{2}, \ldots, \check{h}_{n}\right)=\bigotimes_{j=1}^{n} \check{h}_{\sigma(j)}^{w_{j}} \\
& =\bigcup_{a_{\sigma(i)} \in \check{h}_{\sigma(i)}}\left\{\left(s_{\prod_{j=1}^{n}(\theta(\sigma(j)))^{w_{j}},}\right.\right. \\
& \bigcup_{\left(\mu_{\sigma(i)}, \nu_{\sigma(i)}\right) \in \operatorname{lh}\left(s_{\left.\theta_{\sigma(i)}\right)}\right.}\left(e^{\left.\left.\left.\prod_{j=1}^{n} \ln \mu_{\sigma(j)}^{w_{j}}, 1-e^{\prod_{j=1}^{n}\left(\ln \left(1-v_{\sigma(j)}\right)\right)^{w_{j}}}\right)\right)\right\},}\right.
\end{aligned}
$$

Step 2. Determine the position weight vector $w=\left(w_{1}\right.$, $\left.w_{2}, \ldots, w_{n}\right)$ and the argument weight vector $\omega=\left(\omega_{1}\right.$, $\left.\omega_{2}, \ldots, \omega_{n}\right)$.

Step 3. Utilize the GLHIFHWA (or GLHIFHGM) operator to calculate the collective expected results $\check{h}_{i}$ :

$$
\begin{aligned}
& \check{h}_{i}=\operatorname{GLHIFHWA}\left(\check{h}_{i 1}, \check{h}_{i 2}, \ldots, \check{h}_{i n}\right)=\left(\frac{\bigoplus_{j=1}^{n} w_{j}\left(\omega_{\sigma(j)} \check{h}_{i \sigma(j)}\right)^{\lambda}}{\sum_{j=1}^{n} w_{j} \omega_{\sigma(j)}^{\lambda}}\right)^{1 / \lambda}=\bigcup_{a_{i \sigma(j)}\left(\check{h}_{i \sigma(j)}\right.}\left\{\left(s_{\left(\sum_{j=1}^{n}\left(w_{j}\left(\omega_{\sigma(j)} \theta(i \sigma(j))\right)^{\lambda} / \sum_{j=1}^{n} w_{j} \omega_{\sigma(j)}^{\lambda}\right)\right)^{1 / \lambda},},\right.\right. \\
& \left.\left.\bigcup_{\left(\mu_{i \sigma(j)}, v_{i \sigma(j)}\right) \in l h\left(s_{i \sigma(j)}\right)}\left\{\left(\left(1-\prod_{j=1}^{n}\left(1-\mu_{i \sigma(j)}^{\lambda}\right)^{w_{j} \omega_{\sigma(j)}^{\lambda} / \sum_{j=1}^{n} w_{j} \omega_{\sigma(j)}^{\lambda}}\right)^{1 / \lambda}, 1-\left(1-\prod_{j=1}^{n}\left(1-\left(1-v_{i \sigma(j)}\right)^{\lambda}\right)^{w_{j} \omega_{\sigma(j)}^{\lambda} / \sum_{j=1}^{n} w_{j} \omega_{\sigma(j)}^{\lambda}}\right)^{1 / \lambda}\right)\right\}\right)\right\}, \\
& \check{h}_{i}=\operatorname{GLHIFHGM}\left(\check{h}_{i 1}, \check{h}_{i 2}, \ldots, \check{h}_{i n}\right)=\bigcup_{\left.a_{i \sigma(j)}\right)}\left\{\left(\breve { h } _ { i \sigma ( j ) } \left\{s_{\prod_{j=1}^{n} \theta(i \sigma(j))^{w_{j} \omega_{\sigma(j)} / \sum_{j=1}^{n} w_{j} \omega_{\sigma(j)}},}\right.\right.\right. \\
& \left.\left.\bigcup_{\left(\mu_{i \sigma(j)}, \nu_{i \sigma(j))}\right) \in l h\left(s_{\theta_{i \sigma(j)}}\right)}\left\{\left(1-\left(1-\prod_{j=1}^{n}\left(1-\left(1-\mu_{i \sigma(j)}\right)^{\lambda}\right)^{w_{j} \omega_{\sigma(j)} / \sum_{j=1}^{n} w_{j} \omega_{\sigma(j)}}\right)^{1 / \lambda},\left(1-\prod_{j=1}^{n}\left(1-v_{i \sigma(j)}^{\lambda}\right)^{w_{j} \omega_{\sigma(j)} / \sum_{j=1}^{n} w_{j} \omega_{\sigma(j)}}\right)^{1 / \lambda}\right)\right\}\right)\right\} \text {. }
\end{aligned}
$$


TABLE 1: Linguistic hesitant intuitionistic fuzzy decision matrix.

\begin{tabular}{ccccc}
\hline & $C_{1}$ & $C_{2}$ & $C_{3}$ & $C_{4}$ \\
\hline$A_{1}$ & $\left\{\left(s_{6},(0.5,0.4)\right),\left(s_{7},(0.6,0.3)\right)\right\}$ & $\left\{\left(s_{3},(0.9,0.1),(0.8,0.1)\right)\right\}$ & $\left\{\left(s_{7},(0.7,0.3)\right),\left(s_{8},(0.6,0.2)\right)\right\}$ & $\left\{\left(s_{5},(0.6,0.2)\right)\right\}$ \\
$A_{2}$ & $\left\{\left(s_{3},(0.5,0.2),(0.5,0.3)\right)\right\}$ & $\left\{\left(s_{7},(0.6,0.1)\right),\left(s_{8},(0.8,0.2)\right)\right\}$ & $\left\{\left(s_{6},(0.6,0.3),(0.7,0.2)\right)\right\}$ & $\left\{\left(s_{4},(0.7,0.2)\right),\left(s_{5},(0.5,0.3),(0.6,0.4)\right)\right\}$ \\
$A_{3}$ & $\left\{\left(s_{4},(0.6,0.1)\right)\right\}$ & $\left\{\left(s_{5},(0.7,0.3),(0.5,0.4)\right)\right\}$ & $\left\{\left(s_{2},(0.5,0.4)\right),\left(s_{3},(0.5,0.2)\right)\right\}$ & $\left\{\left(s_{8},(0.6,0.1),(0.7,0.2)\right)\right\}$ \\
$A_{4}$ & $\left\{\left(s_{2},(0.6,0.2),(0.5,0.3)\right)\right\}$ & $\left\{\left(s_{6},(0.8,0.2)\right)\right\}$ & $\left\{\left(s_{3},(0.7,0.2),(0.6,0.3)\right)\right\}$ & $\left\{\left(s_{6},(0.6,0.2)\right),\left(s_{7},(0.7,0.1)\right)\right\}$ \\
$A_{5}\left\{\left(s_{5},(0.7,0.3)\right),\left(s_{6},(0.6,0.2)\right)\right\}$ & $\left\{\left(s_{4},(0.9,0.1),(0.8,0.2)\right)\right\}$ & $\left\{\left(s_{7},(0.8,0.2)\right),\left(s_{8},(0.6,0.4)\right)\right\}$ & $\left\{\left(s_{4},(0.5,0.2)\right)\right\}$ \\
\hline
\end{tabular}

Step 4. Calculate the score function $S\left(\check{h}_{i}\right)$ and accuracy function $A\left(\check{h}_{i}\right)$ of each collective evaluation value $\breve{h}_{i}$

$$
\begin{aligned}
& S\left(\check{h}_{i}\right) \\
& \quad=\frac{1}{l_{\check{h}_{i}}}\left(\sum_{j=1}^{l_{\check{h}_{i}}} \frac{\theta(i j)}{g\left|\operatorname{lh}\left(s_{\theta(i j)}\right)\right|} \sum_{\left(\alpha_{i j}, \beta_{i j}\right) \in \operatorname{lh}\left(s_{\theta(i j)}\right)}\left(\alpha_{i j}-\beta_{i j}\right)\right), \\
& A(\check{h}) \\
& =\frac{1}{l_{\check{h}_{i}}}\left(\sum_{j=1}^{l_{h_{i}}} \frac{\theta(i j)}{g\left|\operatorname{lh}\left(s_{\theta(i j)}\right)\right|} \sum_{\left(\alpha_{i j}, \beta_{i j}\right) \in \operatorname{lh}\left(s_{\theta(i j)}\right)}\left(\alpha_{i j}+\beta_{i j}\right)\right),
\end{aligned}
$$

and here $l_{\check{h}_{i}}$ is the number of LIFEs in $\check{h}_{i},\left|\operatorname{lh}\left(s_{\theta(i j)}\right)\right|$ is the number of intuitionistic fuzzy memberships in $l h\left(s_{\theta(i j)}\right)$, and $g$ is the number of linguistic terms in linguistic term set $S$.

Rank $\check{h}_{i}$ according the method given in Definition 6 and rank the alternatives accordingly.

Step 5. End.

\section{Numerical Examples}

A numerical example is presented to illustrate the efficiency and practical advantages of the proposed procedure.

Consider the decision making problem adapted from [26]. An investor wants to invest some money in an enterprise to get the highest possible profits. He considers five possible alternatives: $\left(A_{1}\right)$ computer company, $\left(A_{2}\right)$ real estate company, $\left(A_{3}\right)$ food company, $\left(A_{4}\right)$ car company, and $\left(A_{5}\right)$ communication company. Multiple experts are invited to evaluate alternatives with respect to the following possible situations for the economic environment: $\left(C_{1}\right)$ negative growth rate, $\left(C_{2}\right)$ low growth rate, $\left(C_{3}\right)$ medium growth rate, and $\left(C_{4}\right)$ high growth rate. The new method is used to rank alternatives.

Step 1. The experts evaluate the alternatives with respect to the attributes using the linguistic arguments and intuitionistic fuzzy memberships and the results are shown in Table 1.

Step 2. The position weight vector is determined as $w=$ $(0.20,0.30,0.35,0.15)$ and the argument weight vector is determined as $\omega=(0.10,0.40,0.15,0.35)$.

Step 3. The GLHIFHSWA operator is used to aggregate the alternatives' evaluation values into collective ones, $\check{h}_{i}(i=1,2, \ldots, 5)$. For example, in order to calculate $\check{h}_{1}$ for $\lambda=0.1$, we first calculate $\omega_{1} \breve{h}_{11}=\left\{\left(s_{0.6},(0.0670,0.9124)\right)\right.$, $\left.\left(s_{0.7},(0.0876,0.8866)\right)\right\}, \omega_{2} \check{h}_{12}=\left\{\left(s_{0.8},(0.3069,0.5253)\right.\right.$, $(0.3822,0.5253))\}, \omega_{3} \breve{h}_{13}=\left\{\left(s_{1.05},(0.1672,0.8348)\right),\left(s_{1.2}\right.\right.$, $(0.1284,0.7855))\}$, and $\omega_{4} \check{h}_{14}=\left\{\left(s_{1.75},(0.2744,0.5693)\right)\right\}$. The scores of $\omega_{j} \check{h}_{1 j}(j=1, \ldots, 4)$ can be calculated as $S\left(\omega_{1} \check{h}_{11}\right)=-0.0593, S\left(\omega_{2} \check{h}_{12}\right)=-0.0161, S\left(\omega_{3} \check{h}_{13}\right)=$ -0.0829 , and $S\left(\omega_{4} \check{h}_{14}\right)=-0.0574$. Then, $\omega_{2} \check{h}_{12}>\omega_{4} \breve{h}_{14}>$ $\omega_{1} \check{h}_{11}>\omega_{3} \check{h}_{13}$. Let $\Phi_{j}=w_{j} \omega_{\sigma(j)}^{0.1} / \sum_{j=1}^{n} w_{j} \omega_{\sigma(j)}^{0.1}$ and $\emptyset_{1}=$ $w_{1} \omega_{\sigma(1)}^{0.1} / \sum_{j=1}^{n} w_{j} \omega_{\sigma(j)}^{0.1}=\left(0.2 * 0.4^{0.1}\right) /\left(0.2 * 0.4^{0.1}+0.3 *\right.$ $\left.0.35^{0.1}+0.35 * 0.1^{0.1}+0.15 * 0.35^{0.1}\right)=0.2135$. We can get $\omega_{2}=0.3160, \omega_{3}=0.3253$, and $\omega_{4}=0.1452$, similarly. By using the GLHIFHSWA ${ }_{01}$ operator, we can get

$$
\begin{aligned}
& \check{h}_{1}=\operatorname{GLHIFHWA}\left(\check{h}_{11}, \check{h}_{12}, \ldots, \check{h}_{14}\right) \\
& =\bigcup_{a_{1 \sigma(j)} \in \check{h}_{1 \sigma(j)}}\left\{\left(s _ { ( 0 . 2 1 3 5 * \theta _ { 1 \sigma ( 1 ) } ^ { 0 . 1 1 } + 0 . 3 1 6 0 * \theta _ { 1 \sigma ( 2 ) } ^ { 0 . 1 } + 0 . 3 2 5 3 * \theta _ { 1 \sigma ( 3 ) } ^ { 0 . 1 } + 0 . 1 4 5 2 * \theta _ { 1 \sigma ( 4 ) } ^ { 0 . 1 } ) ^ { 1 / 0 . 1 } , } \left\{\left(\left(1-\left(1-\mu_{1 \sigma(1)}^{0.1}\right)^{0.2135}\left(1-\mu_{1 \sigma(2)}^{0.1}\right)^{0.3160}\right.\right.\right.\right.\right. \\
& \bigcup_{\left(\mu_{1 \sigma(j)}, v_{1 \sigma(j)}\right) \in \ln \left(s_{\theta_{1 \sigma(j)}}\right)}\left\{\left(1-\mu_{1 \sigma(3)}^{0.1}\right)^{0.3253}\left(1-\mu_{1 \sigma(4)}^{0.1}\right)^{0.1452}\right)^{1 / 0.1}, \\
& 1-\left(1-\left(1-\left(1-v_{1 \sigma(j)}\right)^{0.1}\right)^{0.2135}\left(1-\left(1-v_{1 \sigma(j)}\right)^{0.1}\right)^{0.3160}\right. \\
& \left.\left.\left.\left.\left.\cdot\left(1-\left(1-v_{1 \sigma(j)}\right)^{0.1}\right)^{0.3253}\left(1-\left(1-v_{1 \sigma(j)}\right)^{0.1}\right)^{0.1452}\right)^{1 / \lambda}\right)\right\}\right)\right\} \\
& =\left\{\left(s_{4.2989},\{(0.5689,0.2683),(0.5933,0.2683)\}\right),\right. \\
& \left(s_{4.4945},\{(0.5689,0.2531),(0.5933,0.2531)\}\right), \\
& \left(s_{4.4002},\{(0.6000,0.428),(0.6000,0.2496)\}\right), \\
& \left.\left(s_{4.5999},\{(0.6000,0.2288),(0.6231,0.2288)\}\right)\right\} .
\end{aligned}
$$

Similarly, we can get other collective alternative evaluation values as follows:

$$
\begin{aligned}
\check{h}_{2}= & \left\{\left(s_{5.1977},\{(0.6145,0.2146),(0.6145,0.2404),\right.\right. \\
& (0.6299,0.2146),(0.6299,0.2404)\}),\left(s_{5.4676},\right. \\
& \{(0.6649,0.2759),(0.6649,0.2468),
\end{aligned}
$$$$
(0.6787,0.2468),(0.6787,0.2759)\}),\left(s_{5.3650},\right.
$$

$\{(0.5371,0.2146),(0.5371,0.2404)$, 
TABLE 2: Ranking results based on the GLHIFHSWA operator with different $\lambda$.

\begin{tabular}{lcccccc}
\hline & $S\left(\check{h}_{1}\right)$ & $S\left(\check{h}_{2}\right)$ & $S\left(\check{h}_{3}\right)$ & $S\left(\check{h}_{4}\right)$ & $S\left(\check{h}_{5}\right)$ & Ranking of alternatives \\
\hline$\lambda=0.1$ & 0.1666 & 0.2202 & 0.1915 & 0.2327 & 0.2181 & $A_{4}>A_{2}>A_{5}>A_{3}>A_{1}$ \\
$\lambda=0.2$ & 0.1750 & 0.2239 & 0.1918 & 0.2336 & 0.2192 & $A_{4}>A_{2}>A_{5}>A_{3}>A_{1}$ \\
$\lambda=0.5$ & 0.2025 & 0.2352 & 0.1939 & 0.2357 & 0.2618 & $A_{5}>A_{4}>A_{2}>A_{1}>A_{3}$ \\
$\lambda=1.0$ & 0.2377 & 0.2538 & 0.2018 & 0.2365 & 0.2684 & $A_{5}>A_{2}>A_{1}>A_{4}>A_{3}$ \\
$\lambda=2.0$ & 0.2717 & 0.2864 & 0.2300 & 0.2297 & 0.2853 & $A_{2}>A_{5}>A_{1}>A_{3}>A_{4}$ \\
$\lambda=5.0$ & 0.2875 & 0.3453 & 0.3033 & 0.2065 & 0.3386 & $A_{2}>A_{5}>A_{3}>A_{1}>A_{4}$ \\
$\lambda=10.0$ & 0.2931 & 0.3896 & 0.3497 & 0.1996 & 0.3908 & $A_{5}>A_{2}>A_{3}>A_{1}>A_{4}$ \\
$\lambda=20.0$ & 0.2956 & 0.4132 & 0.3736 & 0.1989 & 0.4212 & $A_{5}>A_{2}>A_{3}>A_{1}>A_{4}$ \\
\hline
\end{tabular}

$$
\begin{aligned}
& (0.5544,0.2404),(0.5544,0.2146)\}),\left(s_{5.6426},\right. \\
& \{(0.5943,0.2468),(0.5943,0.2759), \\
& (0.6102,0.2468),(0.6102,0.2759)\})\}, \\
\check{h}_{3} & =\left\{\left(s_{5.6426},(0.5943,0.2468),(0.5943,0.2759),\right.\right. \\
& (0.6102,0.2468),(0.6102,0.2759)),\left(s_{3.7152},\right. \\
& (0.5994,0.1585),(0.6392,0.2042), \\
& (0.5665,0.1668),(0.6085,0.1370))\}, \\
\check{h}_{4} & =\left\{\left(s_{4.2826},(0.6856,0.1728),(0.6630,0.1946),\right.\right. \\
& (0.6526,0.1996),(0.6283,0.2243)),\left(s_{4.4806},\right. \\
& (0.7002,0.1549),(0.6784,0.1746), \\
& (0.6683,0.1791),(0.6448,0.2016))\}, \\
\check{h}_{5} & =\left\{\left(s_{4.1516},(0.7211,0.1947),(0.6811,0.2243),\right.\right. \\
& (0.6918,0.2164),(0.6486,0.2488)),\left(s_{4.3197},\right. \\
& (0.6543,0.2000),(0.6970,0.1732), \\
& (0.6657,0.1928),(0.6198,0.2222))\} .
\end{aligned}
$$

For other $\lambda$, we can get the results similarly and the results are omitted here for space limit.

Step 4. Calculate the scores $S\left(\check{h}_{i}\right)(i=1,2, \ldots, 5)$ of the comprehensive evaluation values $\check{h}_{i}(i=1,2, \ldots, 5)$ and rank the alternatives according to the ranking of $S\left(\check{h}_{i}\right)(i=$ $1,2, \ldots, 5)$. For $\lambda=0.1$, we can calculate the scores as $S\left(\check{h}_{1}\right)=$ $0.1666, S\left(\check{h}_{2}\right)=0.2202, S\left(\check{h}_{3}\right)=0.1915, S\left(\check{h}_{4}\right)=0.2327$, and $S\left(\check{h}_{5}\right)=0.2181$. The scores can be ranked as $S\left(\check{h}_{4}\right)>S\left(\check{h}_{2}\right)>$ $S\left(\check{h}_{5}\right)>S\left(\check{h}_{3}\right)>S\left(\check{h}_{1}\right)$ and the alternatives can be ranked accordingly as $A_{4}>A_{2}>A_{5}>A_{3}>A_{1}$. For other $\lambda$, we can calculate the scores and rank the alternatives similarly and the results are shown in Table 2.

Step 5. End.
From the above results, we can see that the ranking of alternatives may be different due to different aggregation operators. $A_{4}$ is the optimal alternative if $\lambda=0.1$ or $\lambda=0.2$, since the smallest evaluation values are relatively large in $A_{4}$. The best alternative is $A_{2}$ if the GLHIFHSWA 2 operator and

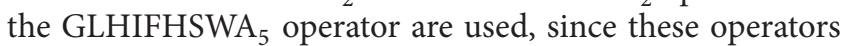
focus on the values with the largest and second largest $\omega_{j}$. $A_{5}$ become the best alternative if the following linguistic hesitant intuitionistic fuzzy aggregation operators are used: the GLHIFHSWA ${ }_{0.5}$ operator, the LHIFHSWA operator, the GLHIFHSWA $_{10}$ operator, and the GLHIFHSWA 20 operator. The GLHIFHSWA ${ }_{0.5}$ operator and the LHIFHSWA operator focus on the average values of the evaluation values. The GLHIFHSWA $_{10}$ operator and the GLHIFHSWA 20 operator focus on the value with the largest weight $\omega_{j}$. With the increasing $\lambda$, the largest weights $\omega_{j}$ play more and more important role in aggregation. $\lambda$ can be seen as the risk attitude of the decision maker. In real decision making problem, decision makers can select the corresponding operator according to real need and his/her interests.

\section{Conclusions}

In this paper, we develop new aggregation operators based on the new fuzzy sets named LHIFSs. Linguistic arguments and intuitionistic fuzzy memberships have been used for evaluation and linguistic hesitant fuzzy information has been gotten, which can reflect the hesitancy, uncertainty, and fuzziness existing in the decision making process. We develop the generalized linguistic hesitant intuitionistic fuzzy hybrid weighted averaging (GLHIFHWA) operator and the generalized linguistic hesitant intuitionistic fuzzy hybrid geometric mean (GLHIFHGM) operator. Some special cases of the new aggregation operators have been studied and many existing aggregation operators are the special cases of the new operators. A new multiple attribute decision making method based on the new operators has been developed and financial product selection problem is presented to illustrate the feasibility and practical advantages of the new methods.

\section{Conflict of Interests}

The authors declare that there is no conflict of interests regarding the publication of this paper. 


\section{Acknowledgments}

The authors would like to thank the editor and reviewers for various helpful and fruitful comments that led to many significant results. This work is partly supported by National Natural Science Foundation of China (nos. 11401457, 11326204, and 61403298) and Shaanxi Province Natural Science Fund of China (nos. 2014JQ1019, 2014JQ8323, and 2014JM1010).

\section{References}

[1] L. A. Zadeh, "Fuzzy sets," Information and Computation, vol. 8, pp. 338-353, 1965.

[2] K. T. Atanassov, "Intuitionistic fuzzy sets," Fuzzy Sets and Systems, vol. 20, no. 1, pp. 87-96, 1986.

[3] K. Atanassov, G. Pasi, and R. Yager, "Intuitionistic fuzzy interpretations of multi-criteria multi-person and multi-measurement tool decision making," International Journal of Systems Science, vol. 36, no. 14, pp. 859-868, 2005.

[4] M. Delgado, J. L. Verdegay, and M. A. Vila, "On aggregation operations of linguistic labels," International Journal of Intelligent Systems, vol. 8, no. 3, pp. 351-370, 1993.

[5] F. Herrera and L. Martínez, "An approach for combining linguistic and numerical information based on the 2-tuple fuzzy linguistic representation model in decision-making," International Journal of Uncertainty, Fuzziness and KnowledgeBased Systems, vol. 8, no. 5, pp. 539-562, 2000.

[6] V. Torra and Y. Narukawa, "On hesitant fuzzy sets and decision," in Proceedings of the 18th IEEE International Conference on Fuzzy Systems, pp. 1378-1382, Jeju Island, Republic of Korea, August 2009.

[7] V. Torra, “Hesitant fuzzy sets," International Journal of Intelligent Systems, vol. 25, no. 6, pp. 529-539, 2010.

[8] M. Xia and Z. Xu, "Hesitant fuzzy information aggregation in decision making," International Journal of Approximate Reasoning, vol. 52, no. 3, pp. 395-407, 2011.

[9] B. Zhu, Z. Xu, and M. Xia, "Hesitant fuzzy geometric Bonferroni means," Information Sciences, vol. 205, pp. 72-85, 2012.

[10] G. W. Wei, "Hesitant fuzzy prioritized operators and their application to multiple attribute decision making," KnowledgeBased Systems, vol. 31, pp. 176-182, 2012.

[11] Z. Zhang, C. Wang, D. Tian, and K. Li, "Induced generalized hesitant fuzzy operators and their application to multiple attribute group decision making," Computers and Industrial Engineering, vol. 67, no. 1, pp. 116-138, 2014.

[12] C. Q. Tan, W. T. Yi, and X. H. Chen, "Hesitant fuzzy Hamacher aggregation operators for multicriteria decision making," Applied Soft Computing, vol. 26, pp. 325-349, 2015.

[13] Z. M. Zhang, "Hesitant fuzzy power aggregation operators and their application to multiple attribute group decision making," Information Sciences, vol. 234, pp. 150-181, 2013.

[14] Z. S. Xu and M. M. Xia, "On distance and correlation measures of hesitant fuzzy information," International Journal of Intelligent Systems, vol. 26, no. 5, pp. 410-425, 2011.

[15] B. Farhadinia, "Information measures for hesitant fuzzy sets and interval-valued hesitant fuzzy sets," Information Sciences, vol. 240, pp. 129-144, 2013.

[16] J. Ye, "Correlation coefficient of dual hesitant fuzzy sets and its application to multiple attribute decision making," Applied Mathematical Modelling, vol. 38, no. 2, pp. 659-666, 2014.
[17] N. Chen and Z. S. Xu, "Hesitant fuzzy ELECTRE II approach: a new way to handle multi-criteria decision making problems," Information Sciences, vol. 292, pp. 175-197, 2015.

[18] N. Zhang and G. Wei, "Extension of VIKOR method for decision making problem based on hesitant fuzzy set," Applied Mathematical Modelling, vol. 37, no. 7, pp. 4938-4947, 2013.

[19] G. Wei, X. Zhao, and R. Lin, "Some hesitant interval-valued fuzzy aggregation operators and their applications to multiple attribute decision making," Knowledge-Based Systems, vol. 46, pp. 43-53, 2013.

[20] X. Zhao, R. Lin, and G. Wei, "Hesitant triangular fuzzy information aggregation based on Einstein operations and their application to multiple attribute decision making," Expert Systems with Applications, vol. 41, no. 4, pp. 1086-1094, 2014.

[21] R. M. Rodríguez, L. Martínez, and F. Herrera, "Hesitant fuzzy linguistic term sets for decision making," IEEE Transactions on Fuzzy Systems, vol. 20, no. 1, pp. 109-119, 2012.

[22] J. Q. Wang, Q. H. Chen, H. Y. Zhang, and X. H. Chen, "An outranking approach for multi-criteria decision-making with hesitant fuzzy linguistic term sets," Information Sciences, vol. 280, pp. 338-351, 2014.

[23] S. M. Chen and J. A. Hong, "Multicriteria linguistic decision making based on hesitant fuzzy linguistic term sets and the aggregation of fuzzy sets," Information Sciences, vol. 286, pp. 6374, 2014.

[24] Z. S. Xu, "Uncertain linguistic aggregation operators based approach to multiple attribute group decision making under uncertain linguistic environment," Information Sciences, vol. 168, no. 1-4, pp. 171-184, 2004.

[25] Z. Zhang and C. Wu, "Hesitant fuzzy linguistic aggregation operators and their applications to multiple attribute group decision making," Journal of Intelligent \& Fuzzy Systems, vol. 26, no. 5, pp. 2185-2202, 2014.

[26] J. M. Merigó and M. Casanovas, "The uncertain induced quasiarithmetic OWA operator," International Journal of Intelligent Systems, vol. 26, no. 1, pp. 1-24, 2011. 


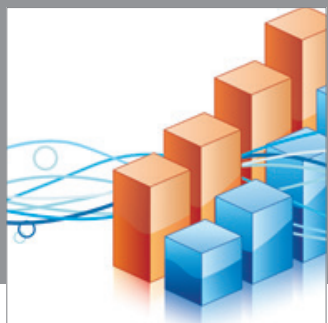

Advances in

Operations Research

mansans

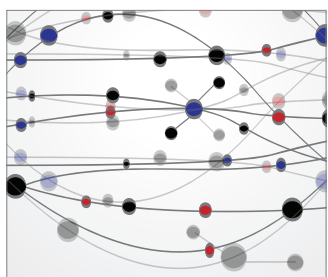

The Scientific World Journal
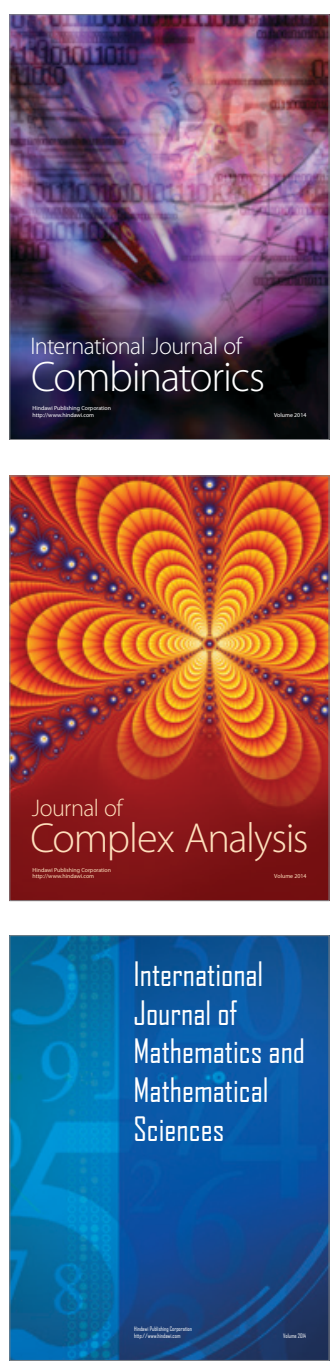
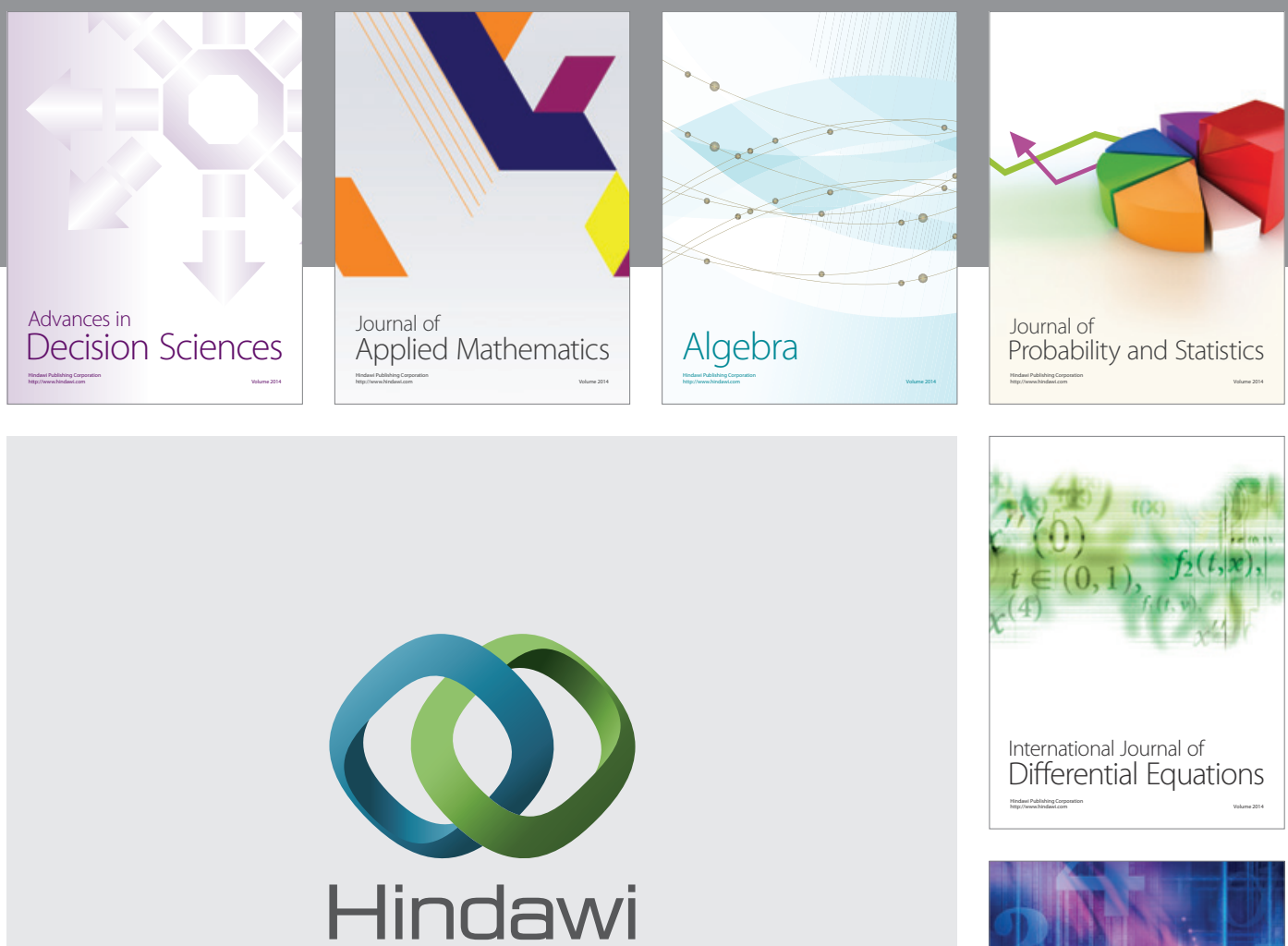

Submit your manuscripts at http://www.hindawi.com
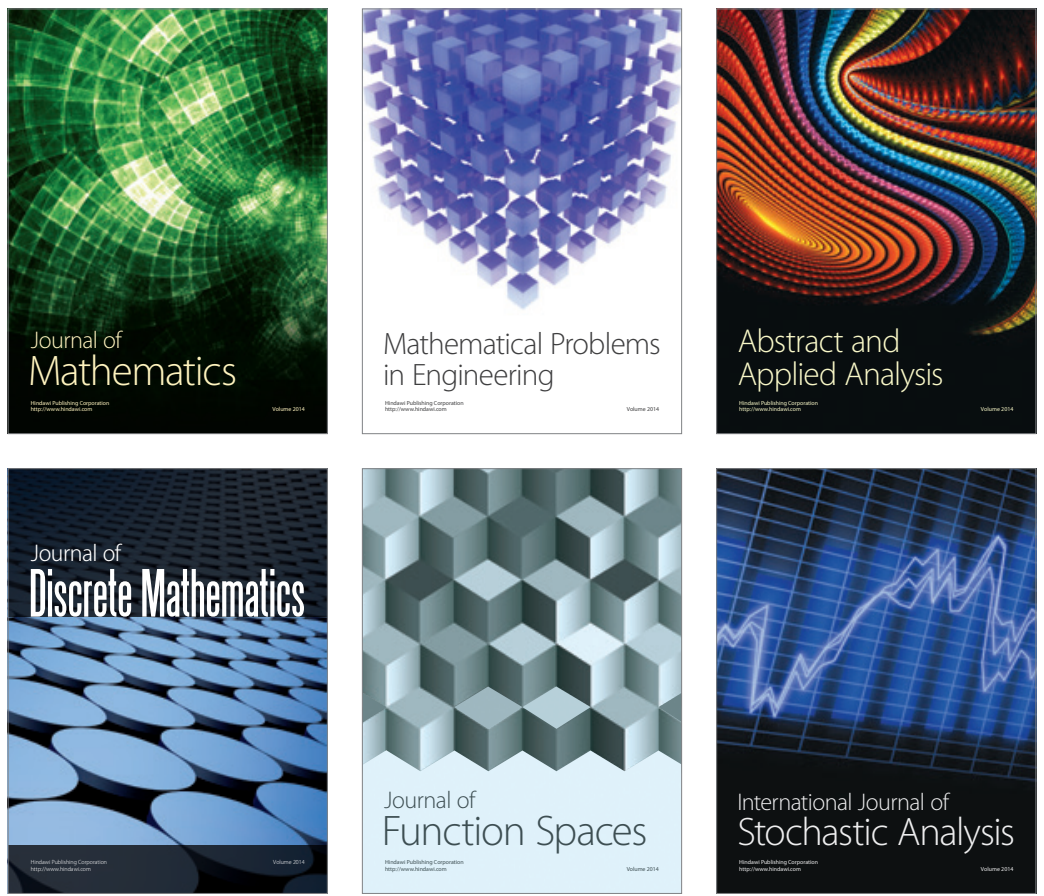

Journal of

Function Spaces

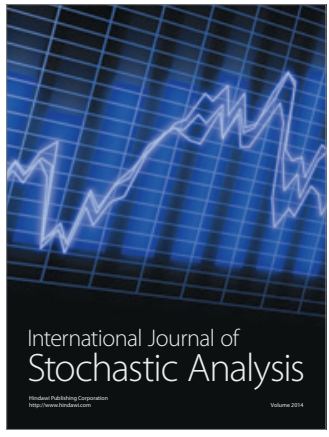

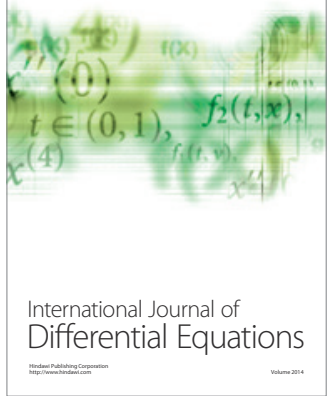
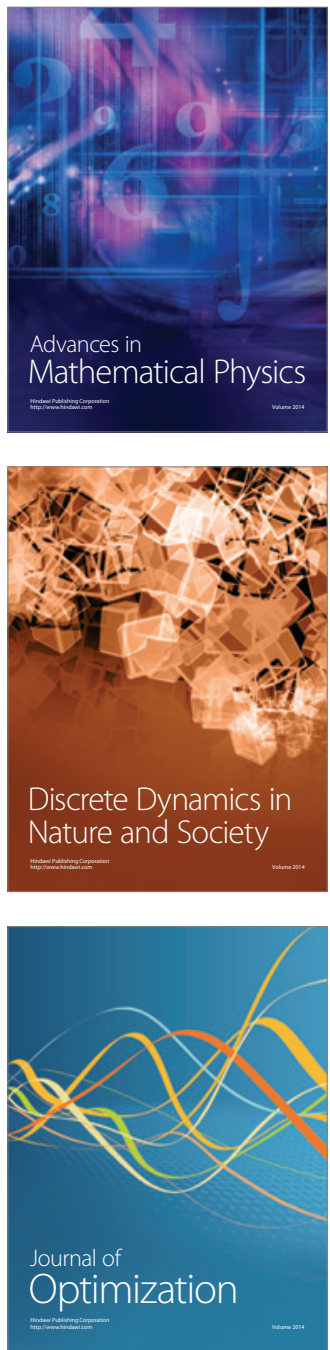